\title{
Integração entre analógico e digital na Arquitetura
}

\author{
- Frederick Gorsten Schunemann \\ Universidade Estadual de Campinas, Brasil \\ fred.gorsten@gmail.com \\ - Gabriela Celani \\ Universidade Estadual de Campinas, Brasil \\ celani@fec.unicamp.br
}

Integration between analog and digital in Architecture

\begin{abstract}
Contemporary architecture relies heavily on high-technology, high-precision methods, such as parametric design and digital fabrication. However, often times architects need to work with preexisting shapes and structures, which are not only irregular but also ephemeral. This paper describes a design exercise in which a bamboo structure was digitized and had a roof system parametrically modeled and fabricated for it.
\end{abstract}

Keywords: Fabricação Digital, Integração Analógico-digital, Modelagem Paramétrica, Prototipagem Rápida

\section{Introdução}

A arquitetura contemporânea caracteriza-se pelo crescente uso de técnicas digitais e computacionais tanto no projeto como na construção. No entanto, esta arquitetura normalmente não referencia diretamente estruturas e/ou elementos arquitetônicos pré-existentes, sendo, em geral, integralmente criada no ambiente digital. A utilização de materiais naturais cujas características são bastante variáveis também não é comum na arquitetura e construção e a compatibilizá-los com métodos construtivos cujo uso é crescente na arquitetura contemporânea é outro campo a ser explorado. O objetivo da pesquisa é investigar a integração entre formas irregulare pré-existentes e reais (analógicas) e as técnicas de modelagem geométrica digital e fabricação por controle numérico. 0 método escolhido foi a pesquisa exploratória, por meio de um exercício de aplicação, já que a investigação prática esclarece quais as possibilidades e dificuldades do processo. As etapas da pesquisa foram:

1.a construção de um modelo utilizando materiais naturais - no caso o bambu;

2.a digitalização 3D por meio de equipamento de toque;

3.a modelagem paramétrica de formas complementares ao modelo digitalizado;

4.a fabricação dessas formas com equipamentos de controle numérico; e

5.a análise retrospectiva do processo de projeto e construção do modelo e a proposição de metodologia para a integração entre fabricação analógica e digital.

\section{Procedimentos Metodológicos}

A pesquisa teve início com uma revisão bibliográfica para fundamentaçãodos conceitos defabricaçãodigital, modelagem paramétrica, equipamentos de controle numérico e processos CAD/CAM, incluindo Barbosa (2012), Kolarevic (2003)e Pupo (2009). Os textos de Celani (2012) foram importantes para entender a aplicação das ferramentas de fabricação digital e prototipagem rápida no contexto brasileiro, tanto no âmbito da indústria da construção como no ensino de arquitetura e urbanismo. Yuan et. al. (2013), Imbern (2014), Varela et. al. e Bonwetsch et. al.(2006) foram referências que guiaram a pesquisa para o tema da integração entre digital e analógico. Os artigos de Zoran (2013) e Muslimin(2010) ressaltaram o potencial de intervir com técnicas de fabricação digital em objetos tradicionais, chamando atenção também para as possibilidades de aplicação dessas técnicas no restauro de elementos arquitetônicos.

O exercício de aplicação envolveu o uso de diferentes tecnologias ligadas ao projeto e construção: digitalização $3 \mathrm{D}$, modelagem paramétrica e a fabricação digital, além da produção manual de uma estrutura. O processo consistiu em inicialmente produzir um modelo em escala reduzida de uma estrutura em bambu, por meio de arcos entrecruzados, produzindo um contraventament, mas com as irregularidades inerentes ao material e a esse método construtivo. Em seguida, a estrutura foi digitalizada com o uso de um equipamento 3D DigitizerMicroScribe G2X. A diferença deste tipo de equipamento com um scanner $3 \mathrm{D}$ é que o primeiro permite 
obter as coordenadas dos pontos desejados, enquanto o segundo gera uma nuvem de pontos com uma quantidade muito grande de coordenadas. Com isso, conseguiu-se a informação suficiente para dar início ao processo de modelagem geométrica de uma cobertura para essa estrutura.

O método empregado foi o da modelagem paramétrica usando o software Grasshopper. Segundo Branco Kolarevic (2003),na modelagem paramétrica não é a forma da geometria que é projetada, mas sim o parâmetro para que ela seja. Isso permitiu um controle maior da modelagem da cobertura e a adaptação às coordenadas dos pontos digitalizados da estrutura de bambu, já que esses pontos foram usados como parâmetros nesse processo. Na modelagem da cobertura foi aplicada uma malha de triângulos, cujos vértices coincidem com as coordenadas dos pontos digitalizados. O material utilizado para a cobertura foi uma lâmina semitransparente flexível cortado por meio de uma plotadora de recorte (vinil cutter), evidenciando a estrutura subjacente.

Para a fixação dos triângulos na estrutura em bambu foram utilizados parafusos e porcas, que criam uma separação entre a estrutura e a cobertura. Essa folga tem a intenção de evitar as irregularidades da superfície do bambu e tensões excessivas no material da cobertura nos pontos de fixação da cobertura à estrutura. No processo de montagem da cobertura, procedeu-se da parte mais baixa para o topo, criando uma estrutura em escamas que confere estanqueidade à água ao modelo.

Além disso, a abordagem paramétrica permitiu que uma substituição de uma peça de bambu na estrutura não gerasse grandes problemas na adaptação do desenho da cobertura, sendo necessária apenas a nova digitalização dos pontos modificados na estrutura e sua referenciação no modelo digital.

\section{Resultados}

O objetivo inicial da pesquisa foi investigar, por meio de um exercício de aplicação, as possibilidades de integração entre produção arquitetônica analógica e digital. Este objetivo foi alcançado, no sentido de entender as dificuldades e os pontos mais determinantes desse processo, além de propor um método específico para adaptação de coberturas a estruturas pré-existentes.

A parte inicial, a montagem da estrutura de bambu, não foi a questão central da pesquisa e sua execução não demandou grandes esforços tanto no projeto da estrutura como na sua confecção.

A etapa da digitalização foi bastante esclarecedora e evidenciou alguns pontos bastante determinantes. $\mathrm{Na}$ primeira tentativa de traduzir a geometria da estrutura de bambu em coordenadas do software utilizado, foram selecionados pontos de número e localização não adequados para o subsequente projeto da cobertura. Por isso, esta etapa foi refeita, sendo obtidas apenas as coordenadas dos pontos em que a cobertura estaria conectada à estrutura. Concluímos que esta etapa tem grande influência no resultado final da integração, já que o excesso ou a falta de pontos digitalizados acarreta problemas ao referenciar estes pontos como parâmetros na etapa de modelagem.

A modelagem paramétrica da cobertura é um processo bastante variável e pode gerar resultados de diferentes graus de complexidade na forma final. Entretanto, independentemente da forma dos elementos modelados, a conexão destes com a estrutura é determinante para que a integração com a estrutura seja consistente. No caso da malha de elementos triangulares utilizada, esses elementos não devem apresentar vértices muito agudos, já que cria pontas excessivamente longas no material da cobertura, dificultando sua fixação à estrutura.

As peças de conexão da cobertura à estrutura também influenciam o processo de montagem. Dependendo da sua forma e do seu mecanismo de fixação, não é possível montar os elementos da cobertura diretamente na posição descrita no projeto, fazendo necessária uma pré-montagem. Para um modelo em escala reduzida isso não é necessariamente um problema, mas em uma escala maior a pré-montagem pode ser inviável. Portanto, procedeu-se no dimensionamento dos elementos da cobertura de modo a não criar elementos de tamanhos muito grandes.

\section{Discussão}

Tendo em vista que este exercício de aplicação (Figura 1) representou o primeiro contato do pesquisador com a modelagem paramétrica, o aprendizado dessa linguagem em si demandou um grande empenho. A linguagem e a estruturação do software requer do modelador um pensamento bastante claro e objetivo, explicitando as relações e interdependências entre os diversos elementos do modelo. A descrição de Robert Woodbury (2010) dessa mudança de paradigma é bastante esclarecedora:

"Conventional design systems are straightforward emulations of this centuries-old means of work. Parametric modeling [...] introduces a fundamental change: 'marks', that is, parts of a design, relate and change together in a coordinated way. No longer must designers simply add and erase. They now add, erase, and repair. The act of relating requires explicit thinking about the kind of relation: is this point on the line, or near to it?Repairing occurs after an erasure, when the parts that depend on an erased part are related again to the parts that remain. Relating and repairing impose fundamental changes on systems and the work that is done with them." 


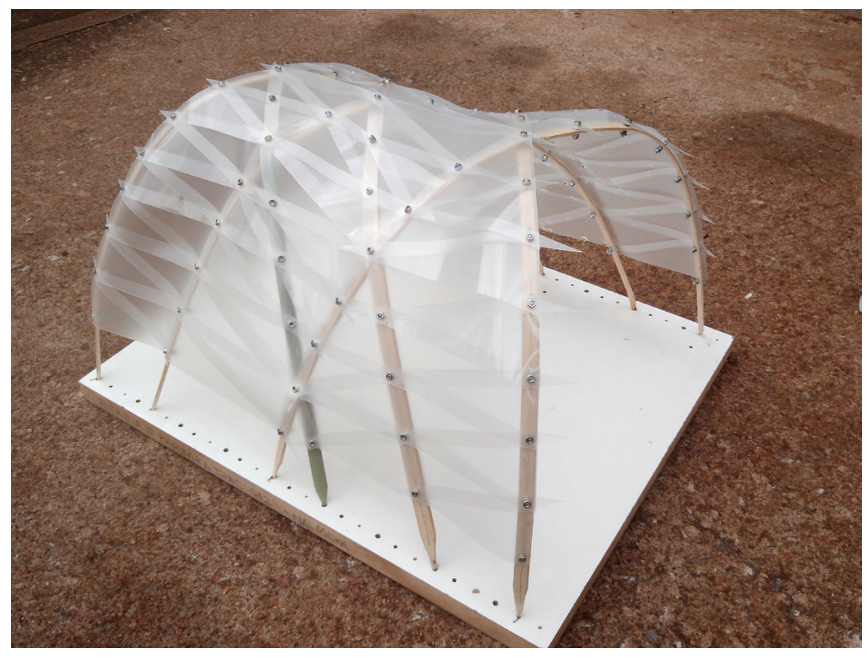

Figura 1: Protótipo construído.

Esse tipo de pensamento não é muito comum no ensino de arquitetura. Os projetos realizados pelos estudantes durante a graduação, quando atingem um grande grau de complexidade geométrica, normalmente não são executados pela falta de instrumentos adequados. A disponibilidade de ferramentas de prototipagem rápida e fabricação digital ganham um papel importante, já que permitem a execução de modelos mais complexos e exigem do projetista um entendimento mais aprofundado e maior controle do processo de modelagem.

Por isso, grande parte do tempo da pesquisa foi dedicado para aprender a dinâmica do software Grasshopper e o funcionamento de suas ferramentas. Esse aprendizado se deu por meio de consulta a diversos vídeos e tutoriais disponíveis na internet, sendo notável o fato de a comunidade de usuários desse software ser bastante ativa e bastante prestativa para ajudar aqueles que estão começando a utilizá-lo.

Tornou-se evidente também a quantidade de empreendimentos e pessoas que utilizam Computer-aided Design (CAD) e Computer-aided Manufacturing (CAM) para produzir os mais variados objetos e quão rápido esse número está se expandindo. Ao buscar na internet informações sobre conceitos ligados a essa nova produção digital, é notável o crescente número de resultados encontrados, sendo a maioria referente a pequenas empresas e escritórios.

No artigo Hybrid (re)Assemblage: An Exploration of Craft, Digital Fabrication and Artifact Uniqueness, de Amit Zoran (2013), sobre o restauro de objetos com uso de modelagem e fabricação digitais notou-se que a integração entre produção analógica e digital já é estudada, mesmo que na escala de objetos menores. A ampliação dessa escala leva também ao restauro arquitetônico, o que é um potencial tema de investigação mais aprofundada e aplicação da integração entre arquitetura analógica e digital.

Com essa pesquisa, espera-se contribuir para a ampliação das possibilidades de aplicação da fabricação digital na arquitetura,criando possibilidades de elaboração de novas formas de concepção e de intervenção em elementos arquitetônicos pré-existentes.

\section{Agradecimentos}

Os autores agradecem ao CNPq pela concessão da bolsa de iniciação científica de Frederick Gorsten Schunemann.

\section{Referências}

Barbosa, W. (2012). Do projeto à fabricação: um estudo de aplicação da Fabricação Digital no processo de produção arquitetônica. Tese de mestrado - Faculdade de Engenharia Civil, Arquitetura e Urbanismo - UNICAMP. Campinas.

Bonwetsch, T., Kobel, D., Gramazio, F., Kohler, M. (2006). The Informed Wall: applying additive digital fabrication techniques on architecture. ETHZ. Zurique. Disponível em: http://cumincad. architexturez.net/system/files/pdf/acadia06_489.content.pdf Celani, G. (2012). Digital Fabrication Laboratories: Pedagogy and Impacts on Architectural Education. Nexus Netw J. 14(3), 469-482. Celani, G. (2012). Chat WithBrankoKolarevic. Parc 4(2), 38-44. Campinas.

Imbern, M. (2014). (Re)Thinking The Brick: Digital Tectonic Masonry Systems. Rethinking Comprehensive Design: Speculative Counterculture, Proceedings of the 19th International Conference on Computer-Aided Architectural Design Research in Asia CAADRIA, 211-220. The Association for Computer-Aided Architectural Design Research in Asia (CAADRIA). Hong Kong. Kolarevic, B. (2003). Architecture in the Digital Age: Design and Manufacturing. Ed. Taylor \& Francis. Londres.

Kolarevic, B. (2001). Designing and manufacturing architecture in the digital age. Architectural information management, 2001117123.

Kolarevic, B., KLINGER, K. (2008). Manufacturing Material Effects: Rethinking Design and Making in Architecture. Ed. Routledge. Londres.

Muslimin, R. (2010). Learning from Weaving for Digital Fabrication in Architecture. Leonardo, 43(4), 340-349. Cambridge, MA: MIT Press.

Pupo, R. T. (2009). Inserção da prototipagem e fabricação digitais no processo de projeto: um novo desafio para o ensino de arquitetura. Tese de doutorado - Faculdade de Engenharia Civil, Arquitetura e Urbanismo - UNICAMP. Campinas.

Varela B., Paio A., Rato V. (2013). Digital tectonic: Rethinking the building with earth in architecture. Vitruvius FabLab-IUL, Instituto Universitário de Lisboa (ISCTE-IUL). Lisboa, Portugal.

Woodburry, Robert (2010). Elements of Parametric Design. Oxon: Routledge.

Yuan, P. F., Zhang, M, Han, L. (2013). Low-tech digital fabrication: Traditional brick as material in digital practice. Proceedings of CAAD Futures 2013.

Zoran, A. (2013). Hybrid Reassemblage: An Exploration of Craft, Digital Fabrication and Artifact Uniqueness. Leonardo, 46(1), 4-10. Cambridge, MA: MIT Press.

Zoran, A. (2013). Hybrid Basketry: Interweaving Digital Practice within Contemporary Craft. Leonardo, 46(4),324-331. Cambridge, MA: MIT Press. 